\title{
Differentiation of Aspartic and Isoaspartic Acids Using Electron Transfer Dissociation
}

\author{
Peter B. O'Connor and Jason J. Cournoyer \\ Boston University Mass Spectrometry Resource, Department of Biochemistry, Boston University \\ School of Medicine, Boston, Massachusetts, USA
}

\author{
Sharon J. Pitteri, Paul A. Chrisman, and Scott A. McLuckey \\ Department of Chemistry, Purdue University, West Lafayette, Indiana, USA
}

\begin{abstract}
Electron-transfer dissociation allows differentiation of isoaspartic acid and aspartic acid residues using the same $c+57$ and $z-57$ peaks that were previously observed with electron capture dissociation. These peaks clearly define both the presence and the position of isoaspartic acid residues and they are relatively abundant. The lower resolution of the ion trap instrument makes detection of the aspartic acid residue's diagnostic peak difficult because of interference with side-chain fragment ions from arginine residues, but the aspartic acid residues are still clearly observed in the backbone cleavages and can be inferred from the absence of the isoaspartic acid diagnostic ions. (J Am Soc Mass Spectrom 2006, 17, 15-19) (C) 2005 American Society for Mass Spectrometry
\end{abstract}

$\mathrm{N}$ onenzymatic deamidation of asparagine, and at a much lower rate glutamine, residues in proteins occurs spontaneously over time with reaction half-lives that range from $\sim 1$ day to $>3$ years, depending on neighboring amino acid residues and on the higher order folding structure of the protein [1]. Deamidation typically proceeds via $\mathrm{SN}_{2}$ attack by the backbone amide on the side-chain carbonyl to displace ammonia forming a cyclic succinimide intermediate (Scheme 1) [2]. This intermediate is unstable in aqueous solutions, and because of the symmetry around the nitrogen in the ring, hydration of the succinimide results in formation of a mixture of aspartic acid and isoaspartic acid. In simple peptide studies, the branching ratio of this experiment is usually $\sim 3: 1$ in favor of the isoaspartic acid residue [3]; however, this ratio is unlikely to hold in whole proteins because of conformational constraints.

This exchange has two large implications on protein tertiary structure. First, it exchanges a neutral or slightly basic residue for an acidic one-thus changing the charge and hydrogen bonding structure. Second, if an isoaspartic acid residue is generated, an additional methylene unit is placed in the protein backbone, pushing the adjacent carbonyl carbon and $\alpha$-carbons apart by an additional 1-1.5 $\AA$. In both cases, the local environment of the initial asparagine/glutamine is dis-

Published online December 9, 2005

Address reprint requests to Dr. Peter B. O'Connor, Boston University School of Medicine, Mass Spectrometry Resource, Department of Biochemistry, 715 Albany Street, Boston, MA 2118, USA. E-mail: poconnor@bu.edu rupted, which could have dramatic impact on the folding structure of the protein. This nonenzymatic posttranslational modification is important to protein folding studies, including antibody-based therapeutics, which could lose activity or generate unexpected side activities because of a change in their tertiary structure. Furthermore, deamidation is commonly considered one of the fundamental modes by which protein aging is controlled in vivo as evidenced by the existence and importance of a repair enzyme [4,5].

Detection of deamidation in peptides and proteins is relatively easy using gel electrophoresis because of the $\mathrm{pI}$ change of the protein or using mass spectrometry because of the +0.984 Da mass shift. However, differentiation of the two products, aspartic acid and isoaspartic acid residues, is much harder, because the two isomeric residues are highly similar in reactivity. The currently existing methods include NMR, Edman-based sequencing methods, and antibody-based detection methods. The first two methods are very insensitive, requiring milligram quantities of pure peptides, and the latter relies on antibodies with relatively poor selectivity. One of the best methods for distinguishing aspartic acid from isoaspartic acid in small peptides is use of high-performance liquid chromatography (HPLC). HPLC, however, also requires relatively large concentrations of peptides to generate a signal in a detector (depending highly on the peptide's amino acid composition) and it separates the two peptides without identifying which is which. Use of the protein isoaspartyl methyltransferase enzyme with radiolabeled methyl groups also 


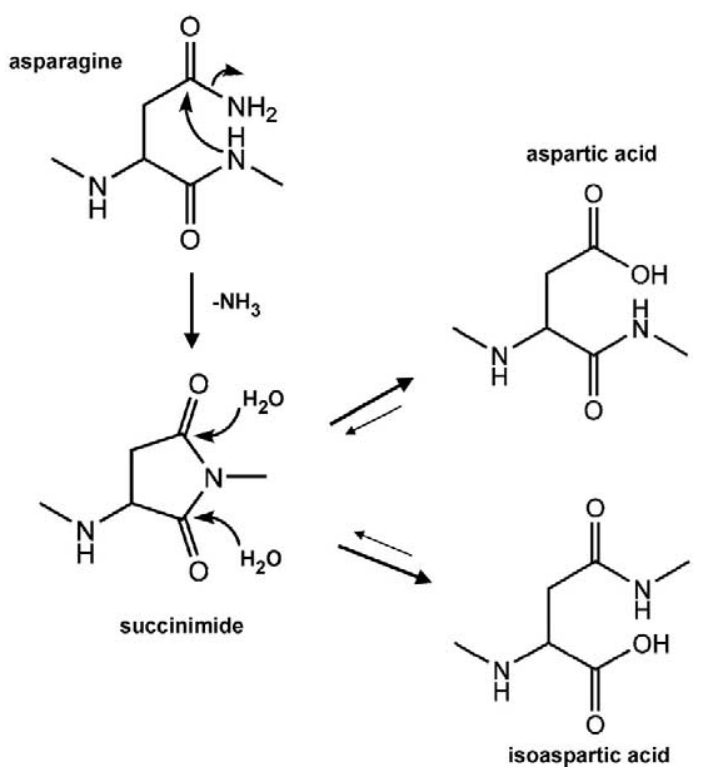

Scheme 1. The standard model mechanism for deamidation of asparaginyl and isomerization of aspartyl residues.

has been used to define the presence (but not the position) of isoaspartic acid [4, 6, 7]. Finally, mass spectrometry has been applied to this problem using traditional collisional activation tandem mass spectrometry methodologies because the relative abundance of the amide bond cleavages next to the aspartic/isoaspartic acid residues can change substantially [8,9]. However, this method has little predictive value unless the peptide in question has been synthesized in both aspartic and isoaspartic acid forms and control spectra have been established before running the unknown.

Electron capture dissociation (ECD), however, primarily cleaves the $n-\mathrm{C}_{\alpha}$ bond in peptides [10]. Because isoaspartic acid does not have an $n-\mathrm{c}_{\alpha}$ bond, it was postulated that the ECD spectra of aspartic acid-containing peptides versus isoaspartic acid containing peptides would be substantially different, allowing facile differentiation of the two residues, a hypothesis that was borne out by experiment [11]. In these experiments, the $c_{\alpha}-c_{\beta}$ bond was cleaved, which generated a diagnostic side-chain cleavage for aspartic acid residues, at M-60 Da and two diagnostic backbone cleavages at $\mathrm{c}_{n}+57\left(\right.$ or $\left.c_{n}^{\bullet}+58\right)$ and $\mathrm{z}_{m}-$ 57 (where $m=$ peptide length $-n$ ). The former determines the presence but not the position of the aspartic acid, but the latter defines both the presence and the position of the isoaspartic acid.

Because electron-transfer dissociation (ETD), [12, 13] like ECD, generates odd-electron fragments and thus proceeds via radical-based rearrangements, here, it is postulated that the same diagnostic fragments observed with ECD will be observed by ETD. This article is intended to report recent results testing this hypothesis.

\section{Methods}

Methanol and glacial acetic acid were purchased from Mallinckrodt (Phillipsburg, NJ). Nitrobenzene was obtained from Sigma-Aldrich (St. Louis, MO). The peptides RAAAGAD $\alpha \mathrm{GD} \alpha \mathrm{GAGAD} \alpha \mathrm{AR}$ and RAAAGAD $\beta$ GD $\beta$ GAGAD $\beta A R$ were synthesized by AnaSpec (San Jose, CA). The peptides were dissolved to $\sim 0.1$ $\mathrm{mg} / \mathrm{mL}$ in $50 / 50 / 1$ ( $\mathrm{vol} / \mathrm{vol} / \mathrm{vol}$ ) methanol/water/ acetic acid for nano-electrospray ionization (ESI) [14, 15]. Nano-ESI was accomplished with nanospray emitters pulled from borosilicate capillaries $(1.5 \mathrm{~mm}$ outer diameter, $0.86 \mathrm{~mm}$ inner diameter) using a P-87 Flaming/Brown micropipet puller (Sutter Instruments, Novato, CA). A stainless steel wire was inserted into the back of the capillary and a potential of $1.5-2 \mathrm{kV}$ was applied to the wire for ionization [16, 17].

All experiments were performed on a Hitachi (San Jose, CA) M-8000 quadrupole ion trap mass spectrometer that has been modified for ion/ion reactions [18]. Nitrobenzene anions were generated using an atmospheric sampling glow discharge ionization [19] source as described previously [20] and were injected into the trap through a hole in the ring electrode. For a typical experiment, peptide cations were injected for $\sim 1 \mathrm{~s}$. Isolation was accomplished using the Hitachi's filtered noise field (FNF) [21, 22] waveforms and by raising the amplitude of the rf signal applied to the ring electrode of the ion trap to eject unwanted ions $(\sim 50 \mathrm{~ms})$. Nitrobenzene anions were injected for $\sim 100 \mathrm{~ms}$ into the ion trap during which time an FNF waveform was applied to eject anions other than nitrobenzene [M $\mathrm{H}]^{-}$and $\mathrm{M}^{-}$ions. Approximately $200 \mathrm{~ms}$ were allowed for ion/ion reactions to occur. After the reaction time, residual anions were ejected by raising the $\mathrm{rf}$ level of the trap, and cations were then analyzed by resonance ejection. Spectra shown here are averaged over $\sim 5 \mathrm{~min}$ ( $\sim 250$ scans). The data were smoothed using five-point boxcar averaging.

\section{Results and Discussion}

Figure 1 shows the ETD spectra of the two model synthetic peptides RAAAGAD $\mathrm{GD}_{\alpha} \mathrm{GAGAD}_{\alpha} \mathrm{AR}$ (Figure 1a) and RAAAGAD ${ }_{\beta} \mathrm{GD}_{\beta} \mathrm{GAGAD}_{\beta} \mathrm{AR}$ (Figure $1 \mathrm{~b}$ ). These tandem mass spectrometry experiments completely sequenced both peptides with all $\mathrm{c}$ and $\mathrm{z}$ ions appearing with $m / z>250$ (except for $z_{11}$, which was present in low abundance but not clearly resolved due to interference from $b_{12}-\mathrm{H}_{2} \mathrm{O}$ ). Cleavages on both sides of the aspartic acid and isoaspartic acid residues at positions 7,9 , and 14 were observed. In addition, a number of $y$ ions as well as the $b_{12}$ and $a_{12}$ ions were apparent in the spectra. For the isoaspartic acid-containing peptide (Figure $1 b$ ), the diagnostic $c_{n}+57$ and $z_{m}-$ $57(m=$ peptide length $-n)$ ions yielded full complementary information on all three isoaspartic acid residue positions. For comparison with prior ECD results [11] it is important to note that the $c_{n}+57$ peak is the 


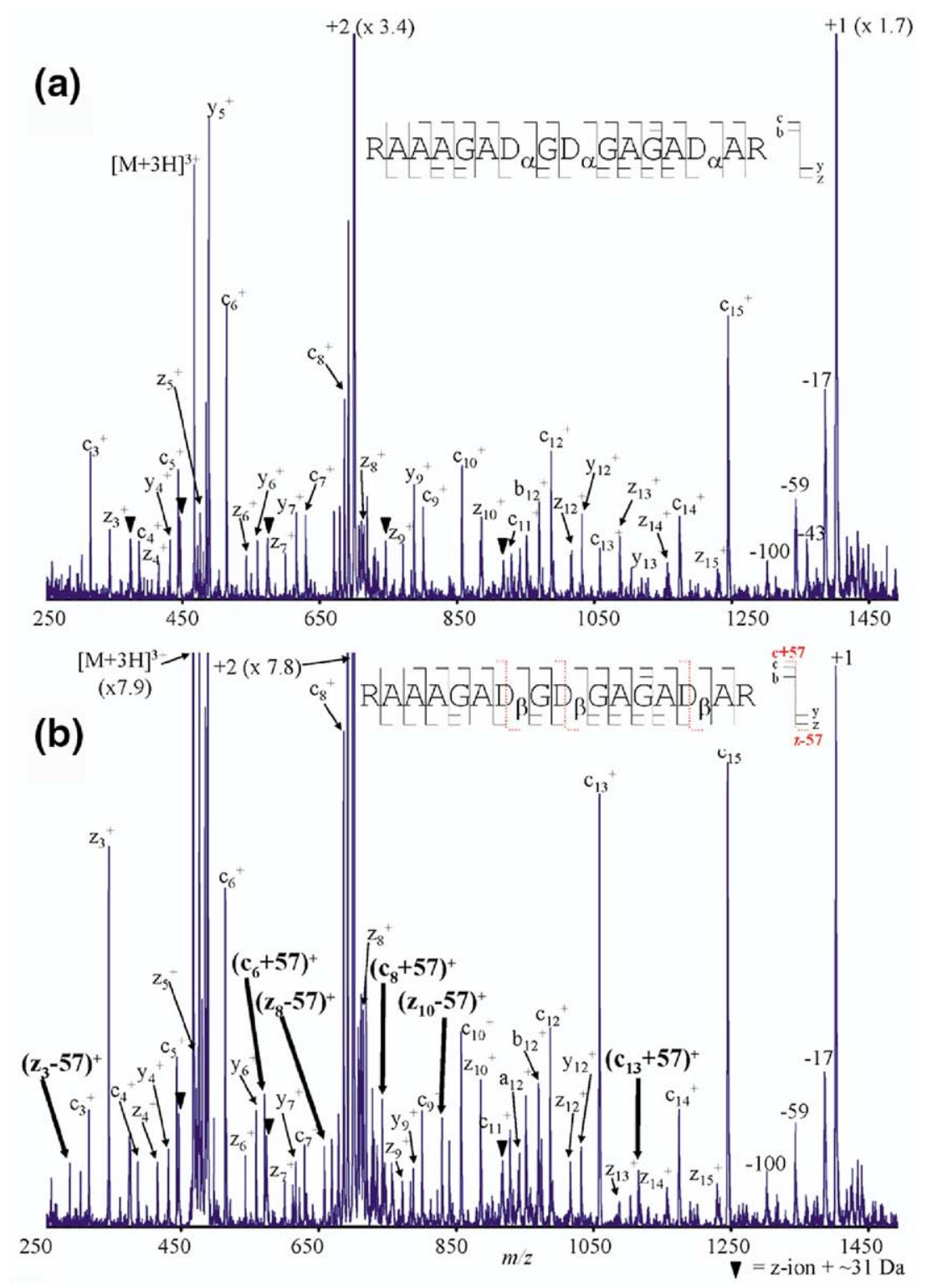

Figure 1. ETD MS/MS spectra of two model synthetic peptides containing (a) aspartic acid and (b) isoaspartic acid residues. Sequence and cleavages are noted on the insets. Bold denotes isoaspartic acid diagnostic ions.

same as the $c_{n}^{\bullet}+58$ peak because a $c_{n}$ ion and a $c_{n}^{\bullet}$ differ by one hydrogen atom. In this case, the $c_{n}+57$ nomenclature is used because the $\mathrm{c}_{n}^{\cdot}$ were not observed, which will be discussed later. The potential observation of the M-60 Da product ion, which is diagnostic of the presence of the aspartic acid residues, was obscured by the M-59 Da arginine side-chain cleavage fragments [23]. Likely, peptides without arginine residues would not experience this interference and the presence of aspartic acid could be reinforced by observation of the M-60 Da ions.

The six isoaspartic acid diagnostic ion mass regions from Figure 1a, b are expanded and shown in Figure 2. For each ion, the left panel shows the data from Figure $\mathrm{lb}$ for the isoaspartic acid-containing peptide and the right panel shows the data from Figure 1a for the aspartic acid-containing analog. The peaks are clear and abundant in the isoaspartic acid containing peptide (left) and are not detectable in the Asp peptide (right), in spite of interference from background $\mathrm{z}+31$ peaks. Clearly, ETD also generates the expected diagnostic ions that had been previously observed by ECD. In Figure 1a, the presence of the aspartic acid residues can be inferred from the absence of the $c+57$ and $z+57$ ions noted previously.

Given the differences in the ECD and ETD experiments, direct comparison of the respective data is complicated. ECD was performed on doubly charged peptide ions whereas ETD was performed on triply charged peptide ions. The different charge states of the peptide likely have different structures and the triply charged ions will at least have an additional protona- 

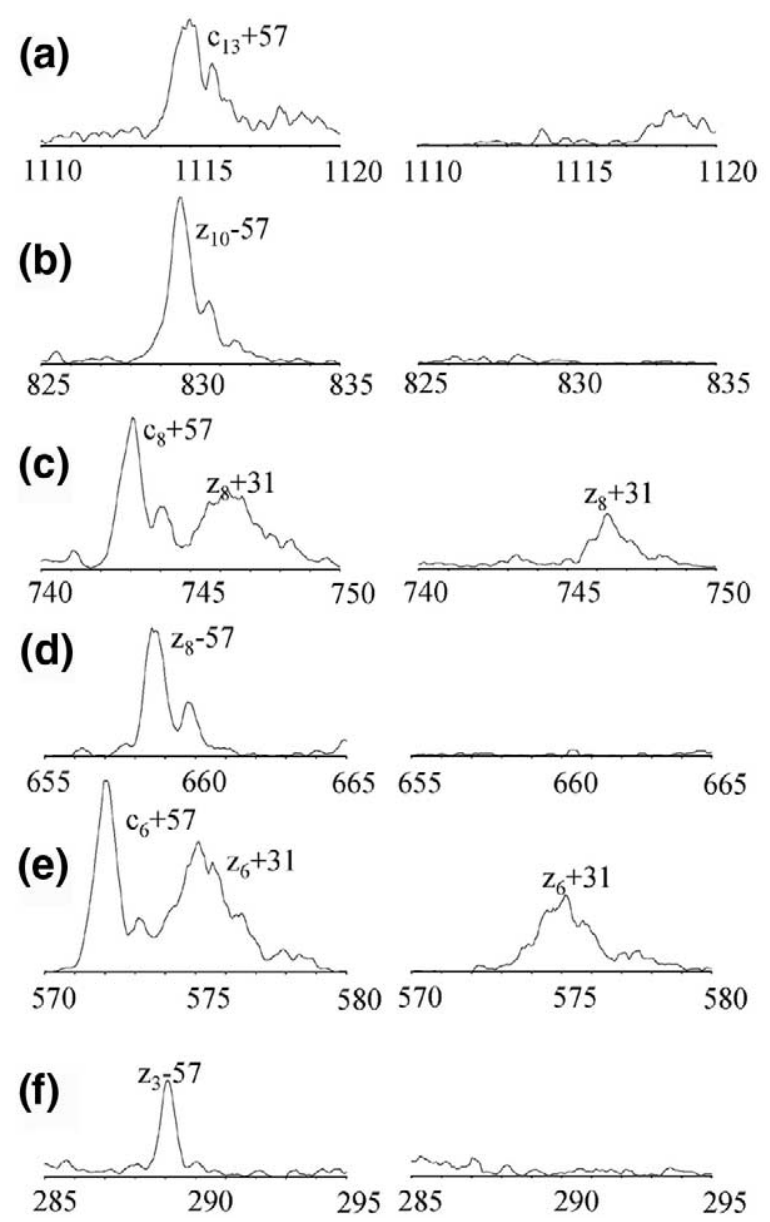

Figure 2. Expanded plots from Figure 1 showing the regions containing the isoaspartic acid diagnostic ions, the left panels are from Figure $1 \mathrm{~b}$ (isoaspartic acid) and the right panels are from Figure 1a (aspartic acid).

tion site, and these factors may well influence the dissociation behavior observed. In addition, the reactions were performed in substantially different environments, with ECD occurring at very low pressure $\left(<10^{-9}\right.$ mbar), and ETD is performed in an ion trap backfilled with helium to a pressure of $\sim 2 \times 10^{-4}$ mbar, which would likely lead to very different activation and cooling rates for the two experiments. Although this makes firm conclusions about any observed differences difficult to draw, comparison of the results of the two experiments yields some interesting observations.

It was noted in the ECD experiments that roughly equal amounts of $\mathrm{c} \bullet / \mathrm{c}$ and $\mathrm{z} \bullet / \mathrm{z}$ product ions were observed for the $\mathrm{N}-\mathrm{C}_{\alpha}$ bond cleavages, whereas the ETD results seemed to show primarily one product ion for each $\mathrm{c}$ or $\mathrm{z}$ ion. Although it is fairly well known that ECD generally tends to produce $\mathrm{c}$ and $\mathrm{z} \bullet$ products, the relative frequency of $\mathrm{c} / \mathrm{z} \bullet$ and $\mathrm{c} \bullet / \mathrm{z}$ formation in ETD still has not been thoroughly investigated. The limited mass accuracy of the ion trap instrument used for these ETD studies makes definitive assignment of these products as even or odd electron species difficult, but it does seem clear that there is primarily one product in each case, not a mixture of the two. Furthermore, the mass difference of 57 between the relevant $c$ ions and the diagnostic $c+57$ ions also suggests that the $c$ ions in those cases are even electron ions.

Also of interest are the $y$ ions, as well as the $b_{12}^{+}$and $\mathrm{a}_{12}^{+}$ions, produced in the ETD experiment. Although possibly these ions are a product of the ETD process, they also may arise by other means. The formation of a and $y$ ions is a well-known minor ECD pathway, which may account for some of these ions, and, in fact, they are observed in the ECD results for this peptide, although the relative frequency of this pathway in ETD still is unknown. The presence of $b$ and $y$ ions could potentially be attributed to various minor collisional activation processes that occur in the ion trap, for example, during the initial cation isolation or during the mass analysis step [18], because cleavage of the $\mathrm{C}_{\mathrm{O}}-\mathrm{N}$ amide bond to produce $\mathrm{b}$ and $\mathrm{y}$ ions is the preferred dissociation pathway for collisional activation of peptide ions. However, the only $b$ ion observed, the $b_{12}^{+}$, is not observed as a major product for collisional activation of the triply or doubly protonated peptide precursor ions (data not shown). In the ECD experiments, prominent $\mathrm{b}$ and $\mathrm{y}$ ions corresponding to cleavage C-terminal to the aspartic acid or isoaspartic acid residues were noted and attributed to collisional activation occurring during the gas pulse before detection or to activation by higher-energy $(9 \mathrm{eV})$ electrons. These ions are not particularly prominent in the ETD results. A final potentially interesting observation from the ETD data is that the $\mathrm{c}$ and $\mathrm{z}$ ions corresponding to cleavage $\mathrm{N}$-terminal of the isoaspartic acid residues appear to increase in relative abundance, when compared with the peptide containing the normal aspartic acid residues. This is particularly noticeable for the $c_{13}^{+}$and $z_{3}^{+}$ ions, but also can be seen in the $\mathrm{c}_{8}^{+}$and $\mathrm{z}_{8}^{+}$ions, and the $\mathrm{c}_{6}^{+}$and $\mathrm{z}_{10}^{+}$ions show no obvious change. A similar change in relative abundance was not noted for the ECD experiments.

The experiments shown here were performed using the $[\mathrm{M}+3 \mathrm{H}]^{3+}$ molecular ions from these model peptides. The ETD reaction with the doubly charged molecular ion showed little or no evidence of dissociation. This is likely because of a shift in the branching ratio between proton transfer (partial neutralization) and electron transfer, with proton transfer becoming the dominant process for the lower-charge states. Such difficulties with ETD of doubly charged peptides have been reported and discussed previously [24].

An intriguing alternative mechanism to explain the limited fragmentation observed in doubly charged ions has been postulated and previously observed on lantibiotics [25]. The ETD reaction will produce two charge reduced molecular ions, the $[\mathrm{M}+2 \mathrm{H}]^{2+}$ or $[\mathrm{M}+3 \mathrm{H}]^{+\bullet}$ ions, as its primary products, which further fragment to produce the observed species. One possible reason for the lack of dissociation products from the $[\mathrm{M}+2 \mathrm{H}]^{2+}$ ion is that the elevated internal vibrational energy available to this radical cation causes extensive secondary fragmenta- 
tion, which distributes the fragment ion intensity into many dissociation channels, preventing detection-similar to the spectra observed in ECD of cyclic peptides [26]. The $[\mathrm{M}+3 \mathrm{H}]^{+} \cdot$, by contrast, has a "quenching" mode available to stabilize the biradical by recombination forming a double-bond or cyclic structure. Thus, backbone fragments could be formed and then stabilized by quenching the biradical. This lantibiotic research showed the presence of both the stabilized and the quenched biradicals using $\mathrm{MS}^{3}$ methods.

The spectra in Figures 1 and 2 show an anomaly, the cause of which is not clear at this time in the form of +31 Da adducts on several of the $z$-ion peaks. These adducts are likely because of reaction of the z-ion radicals with the ETD reagent nitrobenzene or some background neutral molecule. The investigation into the formation of these adducts is ongoing and will be reported separately. Because they are present in both the Asp and the iso-Asp peptide ETD spectra, they have no diagnostic value for distinguishing these residues.

\section{Conclusions}

Differentiation of aspartic and isoaspartic acid residues using ETD is possible using the same $\mathrm{c}+57$ and $\mathrm{z}-57$ peaks that were observed with ECD. These peaks clearly define both the presence and the position of isoaspartic acid residues, are relatively abundant, and often provide complementary information. The lower resolution of the ion trap instrument makes detection of the aspartic acid residue's diagnostic peak difficult because of interference with side-chain fragment ions from arginine residues. Although the $c+57$ and $z-57$ ions peaks are similar between the ECD and ETD reactions (in spite of being performed on completely different instruments at very different background pressures), the observed $\mathrm{b}, \mathrm{y}$, and a ions are quite different. The ETD reaction is not expected to be substantially thermodynamically different from the ECD reaction and actually may be cooler because of the energetics and additional vibrational modes of the anion. However, the presence of abundant $y$ ions and the $b_{12}$ and $a_{12}$ ions indicates that increased vibrational energy is available (compared with ECD) at some point in the experiment. Most likely, this energy arises because of collisional activation of some or all of the reacting species during ion isolation or resonance ejection in the ion trap experiment.

\section{Acknowledgments}

This research was sponsored in part by the National Institutes of Health, National Center for Research Resources (P41RR10888), the National Institutes of Health, National Institute for General Medical Sciences (R01GM45372, to SAM), and from the donors of the American Chemical Society Petroleum Research Fund (to PBO).

\section{References}

1. Robinson, N. E.; Robinson, A. B. Molecular Clocks: Deamidation of Asparaginyl and Glutaminyl Residues in Peptides and Proteins; Althouse Press: Cave Junction, OR, 2004.

2. Radkiewics, J. L.; Zipse, H.; Clarke, S.; Houk, K. N. Accelerated racemization of aspartic acid and asparigine residues via succinimide intermediates: An ab initio theoretical exploration of mechanism. J. Am. Chem. Soc. 1996, 118, 9148-9155.

3. Geiger, T.; Clarke, S. Deamidation, isomerization, and racemization at asparaginyl and aspartyl residues in peptides. J. Biol. Chem. 1987, 262, 785-794.

4. Kim, E.; Lowenson, J. D.; MacLaren, D. C.; Clarke, S.; Young, S. G. The primary structure of a protein carboxyl methyltransferase from bovine brain that selectively methylates l-isoaspartyl sites. J. Biol. Chem. 1997, 94, 6132-6137.

5. Henzel, W. J.; Stults, J. T.; Hsu, C. A.; Aswad, D. W. Deficiency of a protein-repair enzyme results in the accumulation of altered proteins, retardation of growth, and fatal seizures in mice. Proc. Natl. Acad. Sci. U.S.A. 1989, 264, 15905-15911.

6. MacFarlane, D. Inhibitors of cyclic nucleotide phosphodiesterases inhibit protein carboxyl methylation in intact blood platelets. J. Biol. Chem. 1984, 259, 1357-1362.

7. Johnson, B. A.; Aswad, D. W. Enzymatic protein carboxyl methylation at physiological ph: Cyclic imide formation explains rapid methyl turnover. Biochemistry 1985, 24, 2581-2586.

8. Gonzalez, L. J.; Shimizu, T.; Satomi, Y.; Betancourt, L.; Besada, V.; Padron, G.; Orlando, R.; Shirasawa, T.; Shimonishi, Y.; Takao, T. Characterization of aspartic acid and beta-aspartic acid in peptides by fast-atom bombardment mass spectrometry and tandem mass spectrometry. Rapid Commun. Mass Spectrom. 2000, 14, 2092-2102.

9. Castet, S.; Enjalbal, C.; Fulcrand, P.; Guichou, J. F.; Martinez, J.; Aubagnac, J. L. Differentiating alpha- and beta-aspartic acids by electrospray ionization and low-energy tandem mass spectrometry. Rapid Commun. Mass Spectrom. 1996, 10, 1934-1938.

10. Zubarev, R. A.; Kelleher, N. L.; McLafferty, F. W. Electron capture dissociation of multiply charged protein cations-a nonergodic process. J. Am. Chem. Soc. 1998, 120, 3265-3266.

11. Cournoyer, J. J.; Pittman, J. L.; Ivleva, V. B.; Fallows, E.; Waskell, L.; Costello, C. E.; O'Connor, P. B. Deamidation: Differentiation of aspartyl from isoaspartyl products in peptides by electron capture dissociation. Protein Sci. 2005, 14, 452-463.

12. Coon, J. J.; Syka, J. E. P.; Schwartz, J. C.; Shabanowitz, J.; Hunt, D. F. Anion dependence in the partitioning between proton and electron transfer in ion/ion reactions. Int. I. Mass Spectrom. 2004, 236, 33-42.

13. Syka, J. E. P.; Coon, J. J.; Schroeder, M. J.; Shabanowitz, J.; Hunt, D. F. Peptide and protein sequence analysis by electron transfer dissociation mass spectometry. Proc. Natl. Acad. Sci. U.S.A. 2004, 101, $9528-9533$

14. Wilm, M.; Shevchenko, A.; Houthaeve, T.; Breit, S.; Schweigerer, L.; Fotsis, T.; Mann, M. Attomole protein characterization by capillary electrophoresis mass spectometry. Science 1996, 379, 466-469.

15. Valaskovic, G. A.; Kelleher, N. L.; McLafferty, F. W. Femtomole sequencing of proteins from polyacrylamide gels by nano-electrospray mass spectometry. Nature 1996, 273, 1199-1202.

16. Kelleher, N. L.; Senko, M. W.; Siegel, M. M.; McLafferty, F. W. Unit resolution mass spectra of $112 \mathrm{kDa}$ molecules with 3 Da accuracy. J. Am. Soc. Mass Spectrom. 1997, 8, 380-383.

17. Van Berkel, G. J.; Asano, K. G.; Schnier, P. D. Electrochemical processes in a wire-in-a-capillary bulk-loaded, nano-electrospray emitter. J. Am. Soc. Mass Spectrom. 2001, 12, 853-862.

18. Reid, G. E.; Wells, J. M.; Badman, E. R.; McLuckey, S. A. Performance of a quadrupole ion trap mass spectrometer adapted for ion/ion reaction studies. Int. J. Mass Spectrom. 2003, 222, 243-258.

19. McLuckey, S. A.; Glish, G. L.; Asano, K. G.; Grant, B. C. Atmospheric sampling glow discharge ionization source for the determination of trace organic compounds in ambient air. Anal. Chem. 1988, 60, 2220-2227.

20. Hogan, J. M.; Pitteri, S. J.; Chrisman, P. A.; McLuckey, S. A. Complementary structural information from a tryptic n-linked glycopeptide via electron transfer ion/ion reactions and collision-induced dissociation. J. Proteome Res. 2005, 4, 628-632.

21. Goeringer, D. E.; Asano, K. G.; McLuckey, S. A.; Hoekman, D.; Stiller, S. E. Filtered noise field signals for mass-selective accumulation of externally formed ions in a quadrupole ion trap. Anal. Chem. 1994, 66, 313-318.

22. Kelley, P. E. Mass Spectrometry Method Using Notch Filter. 1992

23. Cooper, H. J.; Hudgins, R. R.; Hakansson, K.; Marshall, A. G. Characterization of amino acid side chain losses in electron capture dissociation. J. Am. Soc. Mass Spectrom. 2002, 13, 241-249.

24. Pitteri, S. J.; Chrisman, P. A.; Hogan, J. M.; McLuckey, S. A. Electron transfer ion/ion reactions in a three-dimensional quadrupole ion trap: Reactions of doubly and triply protonated peptides with so_2 $\wedge$. Anal. Chem. 2005, 77, 1831-1839.

25. Heeren, R. M. A.; Kleinnijenhuis, A. J.; Taban, I. M.; Mihalca, R.; Geels, R.; Duursma, M. C.; Heck, A. J. R.; McDonnell, L. A. Does double electron capture lead to the formation of biradicals? An ECD-SORI-CID study on lacticin 481. Presented at the 53rd American Society for Mass Spectrometry Conference, San Antonio, TX, 2005.

26. Leymarie, N.; Costello, C. E.; O'Connor, P. B. Electron capture dissociation initiates a free radical reaction cascade. J. Am. Chem. Soc. 2003, 125, 8949-8958 